\title{
The Great Coronavirus Pandemic of 2019-2021: the Future and the Requirement for China-America Cooperation
}

\author{
Robert C. Gallo*
}

Over the past century, the great pandemics and most epidemics (defined as virus presence and disease induction presenting more than the expected number of infections in a population) were caused by the sudden outbreak of an RNA virus such as the pandemics of influenza, polio, and HIV/AIDS and the epidemics of influenza, Ebola, Dengue, Zika, West Nile, severe acute respiratory syndrome (SARS), Middle East respiratory syndrome (MERS), and Chikungunya. Of course, there are other infections that remain endemic problems in parts of the world, which are caused by bacteria (like tuberculosis) or parasites (like malaria). As everyone knows, the newest and among the most severe pandemic coronavirus disease 2019 (COVID-19) is again caused by an RNA virus, first identified by Chinese medical scientists and shown to be both highly contagious and dangerous (1-4). Why its relatives, SARS and MERS, rather quickly declined and disappeared as a global threat, while COVID-19 became global and persisted unabetted is unknown. What is clear are the following: 1) the Chinese scientific and public health groups such as China CDC were quick and effective for China (see references at the end for a few of the key early papers); 2) the rapid publication (January 10, 2020) of the sequence of the genome of the virus enabled the world to rapidly design vaccine plans and more sophisticated diagnostics; 3) their grasp of transmission by aerosols; 4) asymptomatic persons could be infectious; and 5) their identification of numerous coronaviruses in bats as the key carriers benefited all.

As this virus spread globally, medical scientists were quick to see that it could induce a two-phase disease. First is the establishment of infection and showing mild symptoms, but in many cases progressing to a severe inflammatory disease involving numerous organs but especially lung damage and sometimes leading to death. Progress on developing safe, specific, and potent anti-viral drugs for the early first-stage disease has been slow and disappointing, whereas treatment of the inflammatory stage with dexamethasone has had some significant benefit, but this will soon greatly improve (see below).

As to the future, I am proud to state here that a major new advance will soon be announced from our Institute of Human Virology at the University of Maryland School of Medicine made by Professors Yang Liu and Pan Zheng in close cooperation with the company Oncoimmune. They have used the CD24 molecule to target an inflammatory pathway with specificity and with minimal or no side effects. It has shown outstanding success in COVID-19 patients in all preliminary studies, which will soon be reported. This work began decades ago when Yang Liu came from China to work with Charles Janeway at Yale, one of the greatest basic research immunologists in the world. This dramatic and life-saving result will be reported soon. Of interest, these scientists originating from China are true Chinese-Americans, and they collaborate with Chinese scientists and, of course, with Americans.

We speculate that COVID-19 virus may disappear or be controlled while returning seasonally, similar to the flu, but in reality, we have no idea. We have high hopes for the specific preventive vaccines due to some early positive results, but we must remain vigilant for possibilities of lack of antibody durability, virus escape mutations, and difficulties in global deployment. My collaborators and I have strongly suggested that we keep in mind exploiting the power of innate immunity. This can be achieved by the use of "old" non-specific "live" attenuated vaccines (LAVs) like oral polio, "live" measles vaccines etc., to induce off-target that is nonspecific but powerful induction of innate immunity. Innate immunity responds immediately to invaders and is our first line of everyday defense against infections. Apparently, it is the mechanism used by bats in order to live with their coronavirus infections, and the genome of COVID-19 virus contains regions that have specifically evolved to try to avoid the innate immune system indicating that these viruses are particularly sensitive to these mechanisms protecting us. Clinical studies with COVID-19 virus infection are consistent with this. There is a direct correlation with 
the expression of genes related to innate immune responses and better prognosis, and conversely with bad prognosis when there are deleterious mutations within the innate immunity system. We could have used LAVs at the onset of the pandemic as "stop gaps" until specific vaccines became available. They still can be used if problems occur with the specific vaccines or even with the specific vaccines to enhance them.

Finally, we need better global scientific organization. A "pan" demic means all are affected, and we are all at risk if one member of the global community remains impacted by the virus. All must be together, and that has been far from true so far. I propose that this can best be mediated in part by scientific expertise such as by the deep involvement of the Global Virus Network (GVN), which now consists of 61 Centers of Excellence and 11 Affiliates in 34 countries, containing expertise in virology that covers every type of pathogenic virus and includes animal virology, with a major role for China GVN. During the beginning of the pandemic, we within the GVN had excellent discussions with Chinese colleagues like Yiming Shao and George F. Gao of Beijing as well as Linfa Wang in Singapore, who collaborates closely with Chinese colleagues. It was within the GVN large group discussions that we came to fully realize the importance of innate immunity in controlling COVID-19 virus. Indeed, and above all, we must foster closer ties and collaboration between the US and China, perhaps best mediated by the GVN, which continues to expand its global presence. The world expects this of China and America; the world needs this; the world deserves this. We can accomplish almost anything working together, such as the end of COVID-19 virus and advanced preparation for any future RNA virus threats. Without this, we can be sure such progress has far less chance.

doi: $10.46234 / \mathrm{ccdcw} 2021.037$

\# Corresponding author: Robert C. Gallo, rgallo@ihv.umaryland.edu.

Submitted: January 22, 2021; Accepted: January 29, 2021

\section{REFERENCES}

1. Tan WJ, Zhao X, Ma XJ, Wang WL, Niu PH, Xu WB, et al. Notes from the field: a novel coronavirus genome identified in a cluster of pneumonia cases - Wuhan, China 2019-2020. China CDC Wkly 2020;2(4):61 - 2. http://dx.doi.org/10.46234/ccdcw2020.017.

2. Zhu N, Zhang DY, Wang WL, Li XW, Yang B, Song JD, et al. A novel coronavirus from patients with pneumonia in China, 2019. N Engl J Med 2020;382(8):727 - 33. http://dx.doi.org/10.1056/NEJMoa20010 17.

3. The 2019-nCoV Outbreak Joint Field Epidemiology Investigation Team, Li Q. Notes from the field: an outbreak of NCIP (2019-nCoV) infection in China - Wuhan, Hubei Province, 2019-2020. China CDC Wkly 2020;2(5):79 - 80. http://dx.doi.org/10.46234/ccdcw2020. 022 .

4. Tu WX, Tang HL, Chen FF, Wei YN, Xu TL, Liao KJ, et al. Notes from the field: epidemic update and risk assessment of 2019 novel coronavirus - China, January 28, 2020. China CDC Wkly 2020; 2(6):83 - 6. http://dx.doi.org/10.46234/ccdcw2020.024.

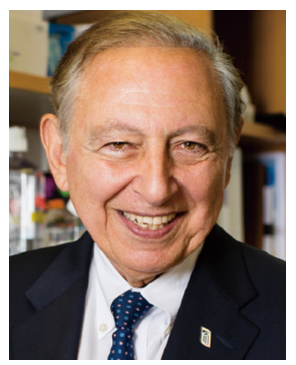

Robert C. Gallo, MD

Gudelsky Distinguished Professor in Medicine and Professor, Microbiology and Immunology

Co-founder and Director, Institute of Human Virology at the University of Maryland School of Medicine, Baltimore, MD, USA

Co-Founder and International Scientific Advisor, Global Virus Network (GVN) 